\title{
INTERVENTIONAL MR-IMAGING FOR THERMAL ABLATION THERAPY
}

\author{
Eva Rothgang** Wesley D. Gilson ${ }^{*} \quad$ Wilhelm Strehl* Li Pan * Jörg Roland ${ }^{\dagger}$ \\ Christine H. Lorenz $z^{\star} \quad$ Joachim Hornegger* \\ * Pattern Recognition Lab, University Erlangen-Nuremberg, Germany \\ * Center for Applied Medical Imaging, Siemens Corporate Research, Baltimore (MD), USA \\ $\dagger$ Siemens Healthcare, Erlangen, Germany
}

\begin{abstract}
Magnetic Resonance Imaging (MRI) has several unique advantages for guiding thermal ablation therapies. It not only provides excellent soft-tissue contrast and multiplanar capabilities, but also is sensitive to thermal effects. To make full use of these advantages for thermal ablation procedures, we present an integrated solution for a thermal therapy workflow that combines dedicated MRI pulse sequences and visualization/analysis tools for trajectory planning, automatic slice positioning for image-guided needle placement, and advanced MR thermal mapping. The paper highlights a novel approach to detect the needle in real-time MR images and to automatically realign the scan planes. In addition, a global approach to correct for $B_{0}$ field shift during online MR thermometry is introduced. The application has been implemented using the open-source eXtensible Imaging Platform (XIP).
\end{abstract}

Index Terms - Magnetic Resonance Imaging, Visualization, Guidance, MR Thermometry

\section{INTRODUCTION}

In modern oncology, thermal ablations are increasingly used as a regional treatment option to supplement systemic treatment strategies such as chemotherapy and immunotherapy $[1,2]$. Current MRI systems are mostly focused on diagnostic imaging and the patient is moved in and out of the scanner for imaging and intervention. Thus, large parts of the MRI workflow have to be customized in order to make intra-procedural MRI guidance and monitoring of thermal therapies feasible in a clinical setting. In the following, the advantages of MRI for interventional guidance of thermal therapies are outlined and it is illustrated how we make use of them in our framework.

First, the excellent soft-tissue contrast of MRI allows very good visualization of the tumor and its margin during the procedure. This is of great importance for both accurate placement of the ablation device and for intraprocedural monitoring. Our dedicated multi-slice, real-time pulse sequences allow us to continuously acquire MR images during all steps of the procedure. The images are sent in real-time over the standard MR scanner network to our application where they are processed for needle guidance or online temperature monitoring.

Second, the multiplanar capabilities of MRI, i.e. the ability to image in three dimensions and in any orientation, allows optimal spatial and temporal guidance of the thermal applicator even into difficult tumor locations. Some proposed approaches for MRI-guided needle placement require additional equipment, for example robots [3], and/or modification of existing equipment, e.g. active needles [4]. In contrast, we employ a passive guidance approach $[5,6]$ working with standard MR-compatible needles. Based on the trajectory planned by the user, we automatically align the MRI slices and detect the device directly from continuously acquired MR images based on exploiting signal voids induced by the device [7].

Third, the linear sensitivity of the MR signal phase to temperature allows for the non-invasive measurement of the spatial and temporal distribution of temperature in vivo [8]. Simultaneously, irreversible tissue necrosis can be delineated in magnitude images because the signal characteristics changes as a result of the change in tissue structure. We make use of this two-fold role of MR in monitoring thermal ablations by calculating temperature maps in real-time and by fusing them with the corresponding magnitude images. Thus, clinicians can monitor the progress of thermal treatment right at the time of the procedure. The reliability of the displayed thermal data is ensured by correcting errors resulting from magnetic field shifts based on a new automatic approach.

\section{METHODS}

The thermal intervention workflow can be divided into three steps, namely planning, targeting, and monitoring (Fig. 1). In the following, we describe how we employ MR imaging to support these steps and what image processing methods we have developed in this context. The presented framework has been implemented using the open-source eXtensible Imaging Platform (XIP) by developing C++ libraries conforming to the Open Inventor standard [9] and is fully integrated into the Interactive Front End (IFE) prototype, a dedicated interactive navigation system for interventional MRI [10]. 


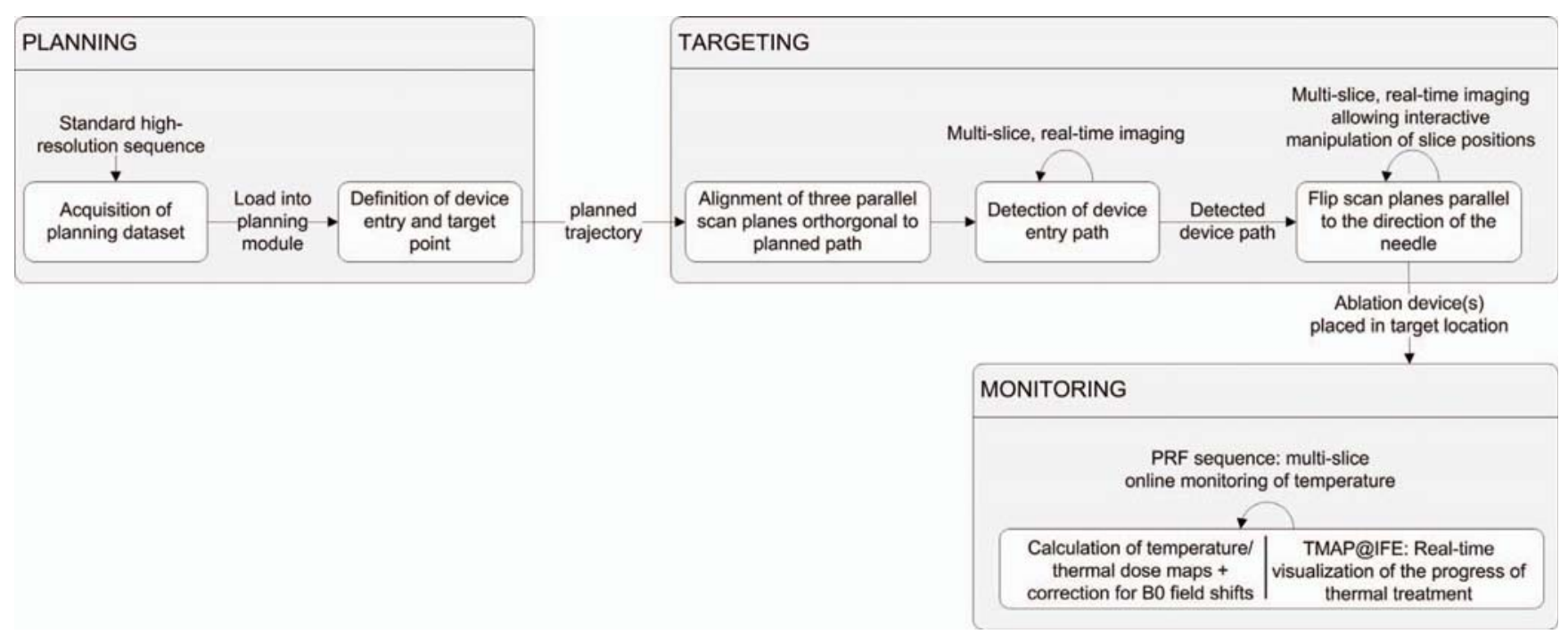

Fig. 1: Proposed workflow for fully MRI-guided thermal ablations combining dedicated MRI pulse sequences and visualization/analysis tools for trajectory planning, automatic slice positioning for image-guided needle placement, and advanced MR thermal mapping.

\subsection{Planning}

MR imaging allows clear delineation of tumor and surrounding tissue mandatory for accurate planning of the ablation procedure. Thus, the first step of our interventional workflow is to acquire a highly resolved dataset using e.g. a fatsuppressed 3D gradient echo sequence. An isotropic or nearisotropic volumetric dataset, can be used for multiplanar reconstruction.

The physician can use it in the planning tool for definition of the device trajectory. The reconstruction of 2-D slices at any arbitrary position and orientation allows the physician to freely navigate through the 3-D dataset in order to decide on an optimal path avoiding vital structures.

For marking the entry point on the patient's skin, the physician is then given the distance required to move the patient table so that the laser light of the MR scanner coincides with the device entry point. In addition, the lateral distance of the device entry point from the laser light is given. The planned trajectory is also used as an input for the following targeting and needle guidance step.

\subsection{Targeting}

For initial needle detection, three parallel scan planes are positioned orthogonal to the planned path at user-defined distances from the entry point. Under continuous imaging, the device entry path is then detected using only image processing methods by exploiting the fact that paramagnetic needles cause a susceptibility artifact in MR images by inducing local field distortions (Fig. 2).

Our needle detection algorithm consists of two steps and

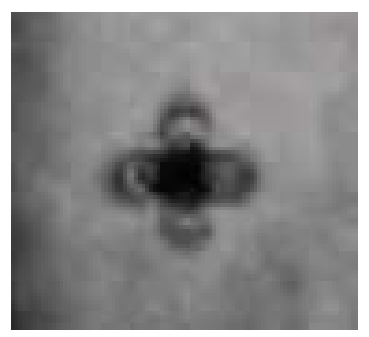

(a) 18G, Cook Medical

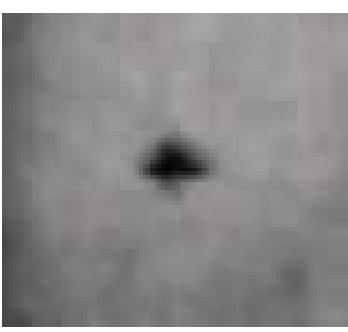

(b) 21G, E-Z-EM, Inc
Fig. 2: The needle artifact is strongly dependent on the needle size and composition. Sequence parameters were the same for both needles which were placed perpendicular to the main magnetic field and imaged orthogonal to the needle length.

is performed independently for each of the three imaging slices. First, the incoming image is compared to a background model to detect regions showing a drop in image intensity. To allow for adaptivity of the background model to small intensity changes, we update the background $R$ by running average. $R_{t}$ is initially set to the first image $I_{0}$ and updated for each pixel $(x, y)$ by

$$
R_{t+1}(x, y)=\alpha R_{t}(x, y)+(1-\alpha) I_{t}(x, y),
$$

where the learning rate $\alpha$ specifies how fast new information supplants old observations. To extract pixels in the incoming image frame exhibiting a significantly lower image intensity compared to the background model, a binary difference image $D_{t}$ is computed as described by

$$
D_{t}(x, y)= \begin{cases}1, & \text { if }\left(R_{t}(x, y)-I_{t}(x, y)\right) \geq \theta \\ 0, & \text { otherwise }\end{cases}
$$


The threshold $\theta$ and $\alpha$ were heuristically set to 50 and 0.25 .

In the second step, connected component labeling [11] is used to extract candidate regions from $D_{t}$. The region is classified as the needle artifact or discarded based on computing various features, such as area and circularity of the region, and proximity to the target path. The circularity can be computed by $C=\frac{P^{2}}{4 A \pi}$, where $A$ is the area of the region and $P$ the perimeter estimated by $P=n_{e}+\sqrt{2} n_{o} . n_{o}$ is the number of odd and $n_{e}$ the number of even chaincodes. The distance of a region's centroid $c$ to the planned trajectory is calculated by $\|p-\phi(c)\|$, where $p$ is the projection of $c$ onto the planned path and $\phi$ is the transformation from the image to the scanner coordinate system. $\|$.$\| denotes the Euclidean length.$

Having detected the device entry path, we orient the scan frames parallel to the direction of the needle to show tumor and applicator simultaneously. We continue real-time imaging for further interactive placement of the applicator into the target tissue and allow the user to interactively adapt the slices positions in 2-D and 3-D views. Further, the target and entry point set by the surgeon in the planning step are marked to provide additional information to anatomical landmarks.

\subsection{Monitoring}

The observation of therapy effects during the ablation procedure is essential in order to achieve the goal of selective tumor treatment without damaging healthy tissue. For monitoring of spatial distribution and temporal evolution of temperature changes during the ablation, we continuously acquire MR magnitude and phase images using a dedicated gradient echo sequence with a standard readout (GRE) or a segmented echo planar imaging readout (GRE-EPI) with a maximum of $10 \mathrm{fps}$. We calculate temperature changes based on the proton resonance frequency method (PRF) [8] which is based on the principle that the phase of the MR signal changes linearly with the temperature of the observed tissue. To extract the phase change of the signal that is proportional to temperature change, baseline images are acquired before the ablation and are then subtracted from intra-therapy phase images based on

$$
\Delta T(x, y, t)=\frac{\phi(x, y, t)-\phi\left(x, y, t_{\text {base }}\right)}{\gamma \alpha B_{0} T_{E}},
$$

where $\gamma=42.58 \mathrm{MHz} /$ Tesla is the gyromagnetic ratio, $\alpha=-0.01 \mathrm{ppm} /{ }^{\circ} \mathrm{C}$ is the temperature sensitivity coefficient, $B_{0}$ is the main magnetic field, $T_{E}$ is the echo time, and $\phi\left(x, y, t_{b a s e}\right)$ is the initial phase before heating. Absolute temperature can then estimated by adding $\Delta T(x, y, t)$ to the user-defined baseline temperature, usually defined as the body temperature $37.2^{\circ} \mathrm{C}$.

From Eq. 3 follows that PRF-derived temperature measurements are sensitive to patient motion and $B_{0}$ drifts. For minimally invasive ablation procedures in the brain, the patient's head is fixed by a stereotactic frame [12] to restrict motion. However, such fixation is not feasible in the abdomen.
Thus, a respiratory-triggered PRF sequence is used to minimize respiratory motion. In both cases, a gradual drift of the $B_{0}$ field occurs resulting in additional phase changes. To account for this effect, the mean phase drift is subtracted from all voxels. It is usually calculated from a manually defined area which is not heated and thus remains at reference temperature. However, it can be difficult to place this region. Our temperature calculation algorithm does not require any user interaction. It determines the mean phase drift from all voxels which show a standard deviation smaller than a threshold $\theta_{\sigma}$ in the phase. For MR thermometry in the brain, we propose a threshold $\theta_{\sigma}$ based on the phase change per ${ }^{\circ} C$, i.e. $\theta_{\sigma}=\gamma \alpha B_{0} T_{E}$. Thus, all voxels having a standard deviation from $37.2^{\circ} \mathrm{C}$ over time smaller than $\theta_{\sigma}$ are included in the calculation of the mean phase drift.

\section{RESULTS}

The experiments were performed on a Siemens Magnetom Avanto 1.5 Tesla MR Scanner and a Siemens Magnetom Espree 1.5 Tesla MR Scanner.

\subsection{Planning and Targeting}

The functionality of the planning tool was tested by using its output, the planned trajectory, as an input for needle detection in the targeting step. In order to measure the accuracy of initial needle detection, and hence alignment of the scan planes for targeting, we designed a fully MR-compatible phantom (Fig. 3). The precisely machined phantom served as the ground truth for needle position and was aligned to the scanner coordinate system by utilizing the laser of the MR scanner. Landmarking with the laser defines a given position on the object that when moved into the magnet is coincident with the isocenter of the magnet (with respect to the scanner coordinate system).

Using the phantom, we biopsied a pork sample 11 times with each needle under continuous imaging at a sampling rate of $5 \mathrm{fps}$ using our interactive, real-time, multi-slice TrueFISP sequence (BEAT_iRTTT). We successfully detected the artifact automatically in all 66 slices. The mean detection accuracy was $1.3 \mathrm{~mm}(\sigma=0.6 \mathrm{~mm})$ for the $18 \mathrm{G}$ needle and $1.6 \mathrm{~mm}$ $(\sigma=0.9 \mathrm{~mm})$ for the $21 \mathrm{G}$ needle. This accuracy is sufficient to realign slice planes for guidance as it is much less than the standard slice thickness $(5-8 \mathrm{~mm})$ used. For our needle detection experiment, we used two MRI compatible biopsy needles (COOK Medical and E-Z-EM, Inc) of size 18G and 21G. Different orientations, needle compositions, pulse sequences and field strengths affect needle artifacts [13]. The effect of needle size and composition on artifact size and shape is illustrated in Figure 2. 

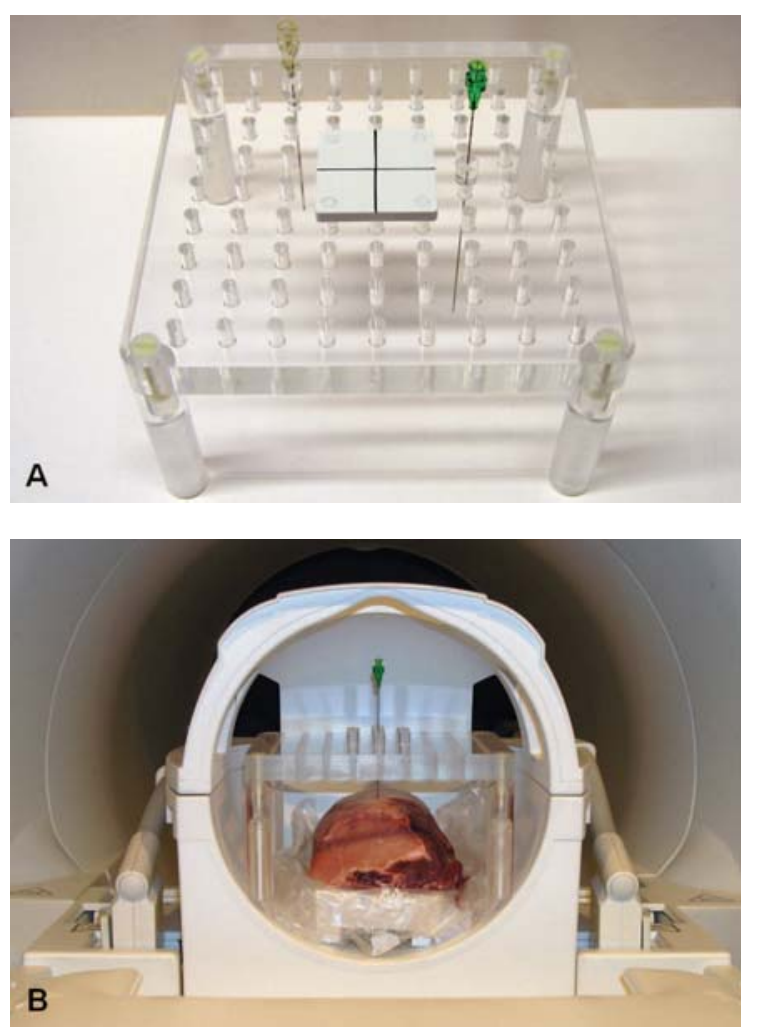

Fig. 3: A. Custom-built, fully MR compatible phantom for validation of needle detection accuracy. The needle pins can be exchanged to exactly fit the used needle. Calibration is performed with the freely placeable white board. B. The phantom is placed inside a standard head coil which we used for our experiments.

\subsection{Monitoring}

As shown in Figure 4, a prototype with advanced visualization tools was created to closely monitor temperature changes during the ablation.

As windowing is extensively used in MRI to highlight tissue boundaries during image interpretation, it should work in the same manner for combined anatomical and thermal images. We created a fused HLS-coded image by mapping the lightness to the magnitude image and the hue to the colorcoded temperature image. For display purposes, we converted the HLS image to RGB (Fig. 4, lower right 2-D view). The HSL-color space was chosen as it is symmetric to lightness and darkness.

As the PRF method has been extensively validated [14], we focus on $B_{0}$ field shift correction and validate the temperature precision in an unheated human volunteer brain study (3 volunteers, 10 measurements). Figure 5 shows the effect of our $B_{0}$ field shift correction algorithm exemplary for a randomly selected voxel. When correcting for this shift, we achieve a temperature accuracy of $0.5^{\circ} \mathrm{C}$ in the brain.

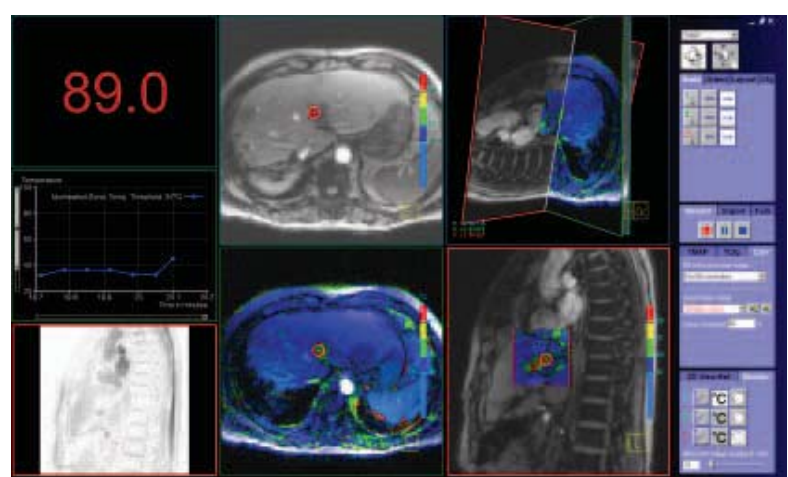

Fig. 4: Screen capture of the advanced real-time visualization application, called TMAP@IFE, for thermal therapy showing a patient data set acquired during a laser-induced thermal ablation in the liver using the dedicated gradient echo PRF sequence with respiratory triggering. On the left, the user can either display a graph showing temperature over time for selected seed points or a difference image. In the 2-D and 3-D views, the acquired slices are visualized in real-time according to the fusion mode set by the user in the control panel on the right.

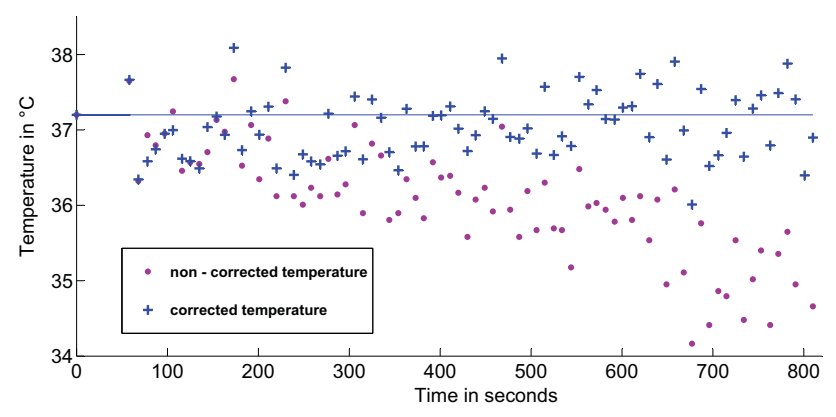

Fig. 5: Automatic $B_{0}$ drift correction and resulting temperature accuracy over time for a randomly selected voxel in the brain. The baseline temperature of $37.2{ }^{\circ} \mathrm{C}$ is given by the blue line.

\section{CONCLUSIONS}

This paper describes several components of an integrated system for planning, guiding and monitoring thermal ablation therapy using MRI. Special focus is put on a novel targeting approach, based on frame differences with temporal background smoothing and candidate extraction, and on a global approach for B0 drift correction.

Initial validation of the individual components and the complete workflow is promising, and the proposed system is anticipated to improve the efficacy and efficiency of thermal ablations. However, more extensive validations of the in vivo needle placement and temperature mapping are needed. 


\section{ACKNOWLEDGMENTS}

We would like to thank the team of the workshop at Siemens MR, Erlangen, for their support in building the phantom. We would also like to thank the team of Prof. Norbert Hosten from the University of Greifswald and Antje Kickhefel from Siemens Healthcare, Erlangen, for providing anonymized LITT patient data sets.

\section{REFERENCES}

[1] S. Tatli, P.R. Morrison, K. Tuncali, and S.G. Silverman, "Interventional MRI for Oncologic Applications," Techniques in Vascular and Interventional Radiology, vol. 10, no. 2, pp. 159-170, 2007.

[2] V. Rieke and K. Butts Pauly, "MR Thermometry," Journal of Magnetic Resoance Imaging, vol. 27, no. 2, pp. 376-390, 2008.

[3] A. Krieger, R.C. Susil, C. Ménard, J.A. Coleman, G. Fichtinger, E. Atalar, and L.L. Whitcomb, "Design of a novel MRI compatible manipulator for image guided prostate interventions," Biomedical Engineering, IEEE Transactions on, vol. 52, no. 2, pp. 306-313, 2005.

[4] S. Sathyanarayana, P. Aksit, A. Arepally, P.V. Karmarkar, M. Solaiyappan, and E. Atalar, "Tracking planar orientations of active MRI needles," Journal of Magnetic Resonance Imaging, vol. 26, no. 2, pp. 386-391, 2007.

[5] S.P. DiMaio, E. Samset, G.S. Fischer, I.I. Iordachita, G. Fichtinger, F.A. Jolesz, and C.M.C. Tempany, "Dynamic MRI scan plane control for passive tracking of instruments and devices," in Lecture Notes in Computer Science, 2007, vol. 4792, pp. 50-58.

[6] A. de Oliveira, J. Rauschenberg, D. Beyersdorff, W. Semmler, and M. Bock, "Automatic passive tracking of an endorectal prostate biopsy device using phase-only cross-correlation," Magnetic Resonance in Medicine, vol. 59, no. 5, pp. 1043-1050, 2008.

[7] M.E. Ladd, P. Erhart, J.F. Debatin, B.J. Romanowski, P. Boesiger, and G.C. McKinnon, "Biopsy needle susceptibility artifacts," Magnetic Resonance in Medicine, vol. 36, no. 4, pp. 646-651, 1996.

[8] Y. Ishihara, A. Calderon, H. Watanabe, K. Okamoto, Y. Suzuki, K. Kuroda, and Y. Suzuki, "A precise and fast temperature mapping using water proton chemical shift," Magnetic Resonance in Medicine, vol. 34, no. 6, pp. 814-823, 1995.

[9] G. Paladini and F.S. Azar, "An extensible imaging platform for optical imaging applications," in Proceedings of SPIE, 2009, vol. 7171, p. 717108.
[10] C.H. Lorenz, K.J. Kirchberg, S. Zuehlsdorff, P. Speier, M. Caylus, W. Borys, T. Moeller, and M.A. Guttman, "Interactive Front End (IFE) - a platform for graphical MR scanner control and scan automation," 2005, Proceedings of ISMRM, p. 2170.

[11] L. Shapiro and G. Stockmann, Computer Vision, Prentice Hall, 2001.

[12] A. Carpentier, R.J. McNichols, R.J. Stafford, J. Itzcovitz, J.P. Guichard, D. Reizine, S. Delaloge, E. Vicaut, D. Payen, A. Gowda, and B. George, "Real-time magnetic resonance-guided laser thermal therapy for focal metastatic brain tumors," Neurosurgery, vol. 63, no. 1, pp. 21-29, 2008.

[13] J.S. Lewin, J.L. Duerk, V.R. Jain, C.A. Petersilge, C.P. Chao, and J.R. Haaga, "Needle localization in MRguided biopsy and aspiration: effects of field strength, sequence design, and magnetic field orientation," American Journal of Roentgenology, vol. 166, no. 6, pp. 1337-1345, 1996.

[14] N. McDannold, “Quantitative MRI-based temperature mapping based on the proton resonant frequency shift: Review of validation studies," International Journal of Hyperthermia, vol. 21, no. 6, pp. 533-546, 2005. 